Supporting information for

\title{
Bio-Inspired Photonic Masquerade with Perturbative Metasurfaces
}

\author{
Kun Huang ${ }^{1,2, *}$, Dong Zhao ${ }^{1}$, Febiana Tjiptoharsono ${ }^{2}$, Yunjie $\mathrm{Chen}^{2}$, Calvin Pei Yu \\ Wong $^{2}$, Xiaosong Tang ${ }^{2}$, Joel K. W. Yang ${ }^{2,3}$, and Zhaogang Dong, ${ }^{2, *}$
}

\begin{abstract}
Affiliations:
${ }^{1}$ Department of Optics and Optical Engineering, University of Science and Technology of China, Hefei, Anhui 230026, China

${ }^{2}$ Institute of Materials Research and Engineering, Agency for Science, Technology and Research (A*STAR), 2 Fusionopolis Way, \#08-03, Innovis, Singapore 138634

${ }^{3}$ Singapore University of Technology and Design, 8 Somapah Road, Singapore 487372, Singapore

*To whom correspondence should be addressed. E-mail: huangk17@ustc.edu.cn, dongz@imre.a-star.edu.sg
\end{abstract}




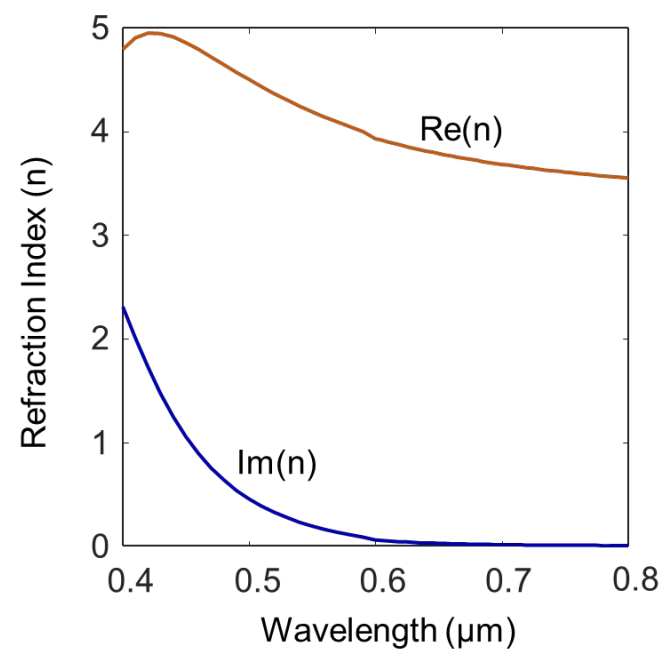

Figure S1. The experimentally measured refraction index of a $305 \mathrm{~nm}$-thick Si film on the quartz substrate by using an ellipsometer.
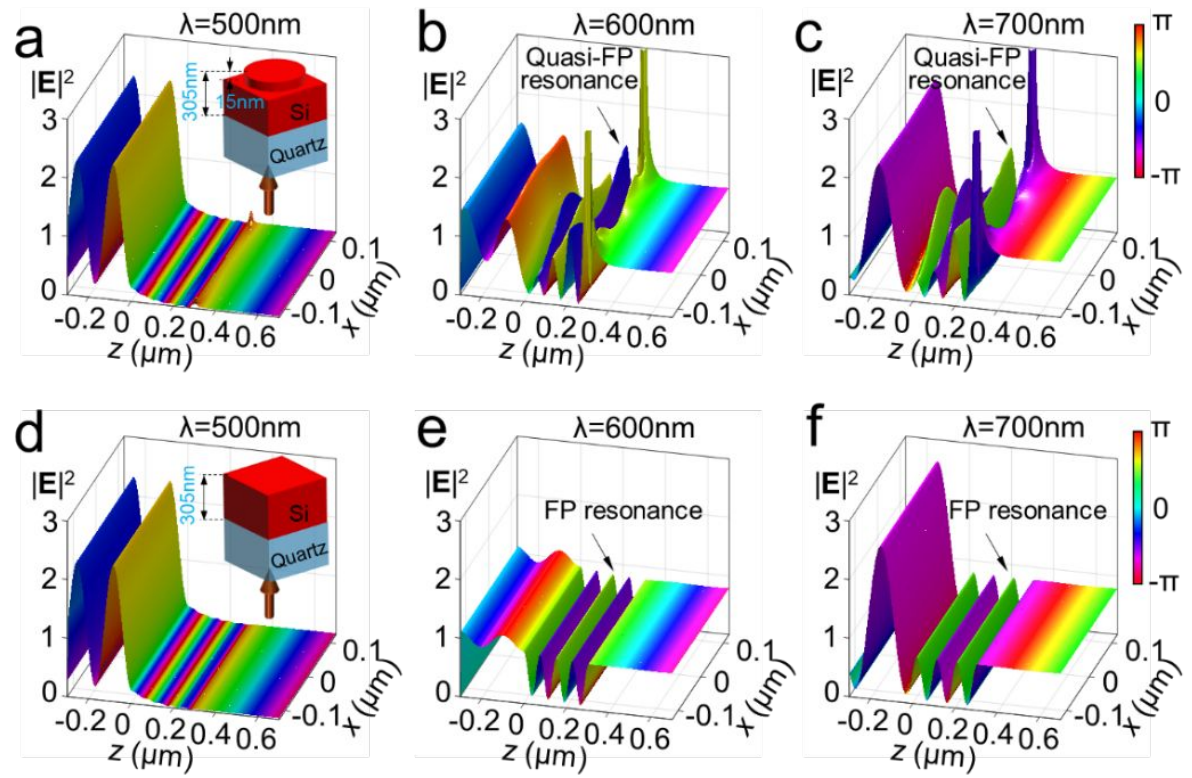

Figure S2. Simulated electric fields in meta-structures (a-c) and Si film (d-f) at three typical wavelengths of $500 \mathrm{~nm}, 600 \mathrm{~nm}$ and $700 \mathrm{~nm}$. For both cases, the interface between quartz and Si film is located at $z=0$. At the region $z=0$ to $z=305 \mathrm{~nm}$, the electric field in Si oscillates faster than those in $\operatorname{air}(z>305 \mathrm{~nm})$ and quartz $(z<0)$. For the metastructure with $h=15 \mathrm{~nm}$, one can find that the electric fields (500 nm (a), $600 \mathrm{~nm}$ (b) and $700 \mathrm{~nm}$ (c)) in Si exhibit the quasi-Fabry-Perot (FP) resonances, which behaves like the cases of Si film (i.e., object). So, their transmission and phase modulation are highly consistent, enabling the optical masquerade. We note that, the electric fields at the gap of two nanodisks is strong due to the enhancement effect of field, which is also predicted in plasmonic systems. 

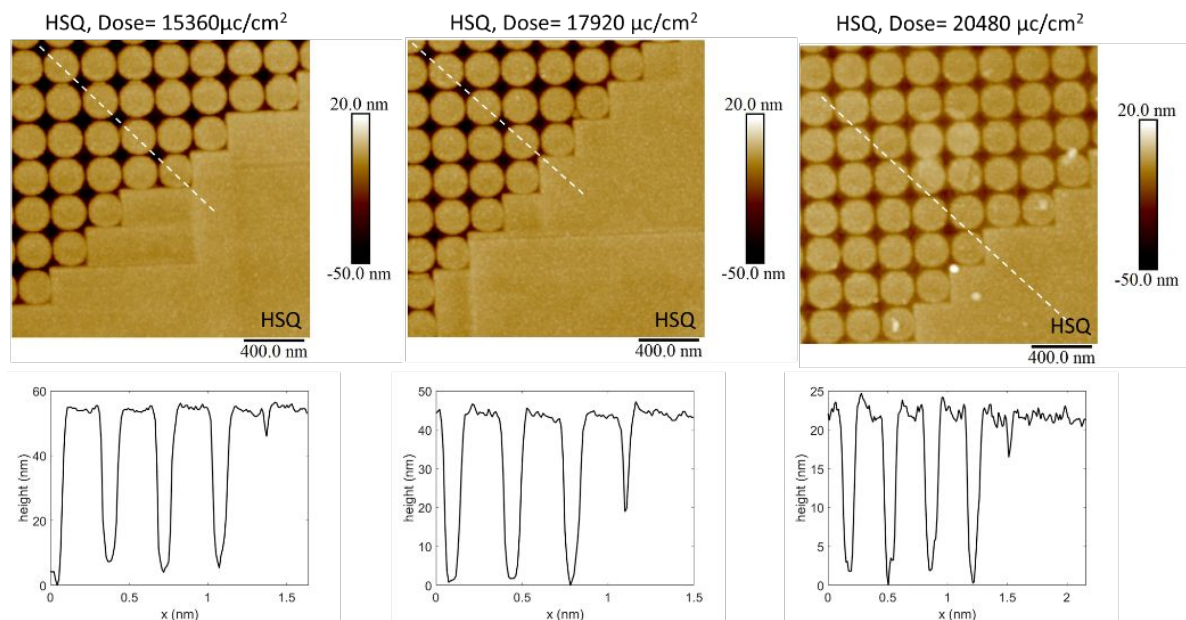

Figure S3. AFM images of photoresist (HSQ) after development when the e-beam dosage is different. The large dosage of e-beam leads to the strong back scattering of electrons so that the surrounding HSQ beyond the patterned area is also exposed, leading to a shallow HSQ, which can be observed by the line-scanning profiles of AFM images. The depth of HSQ after development changes from $50 \mathrm{~nm}, 40 \mathrm{~nm}$, to $20 \mathrm{~nm}$ for the e-beam dosage of $15360 \mu \mathrm{c} / \mathrm{cm}^{2}, 17920 \mu \mathrm{c} / \mathrm{cm}^{2}$ and $20480 \mu \mathrm{c} / \mathrm{cm}^{2}$, respectively.

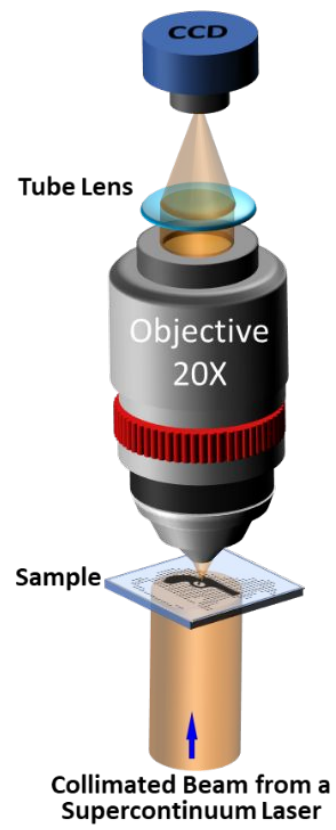

Figure S4. Experimental setup for characterizing optical masquerade. A collimated beam from a supercontinuum laser is used to illuminate the sample through the substrate. The transmitted light is collected by a 20X-magnification objective (ZEISS) and then projected onto a CCD camera by a tube lens. 

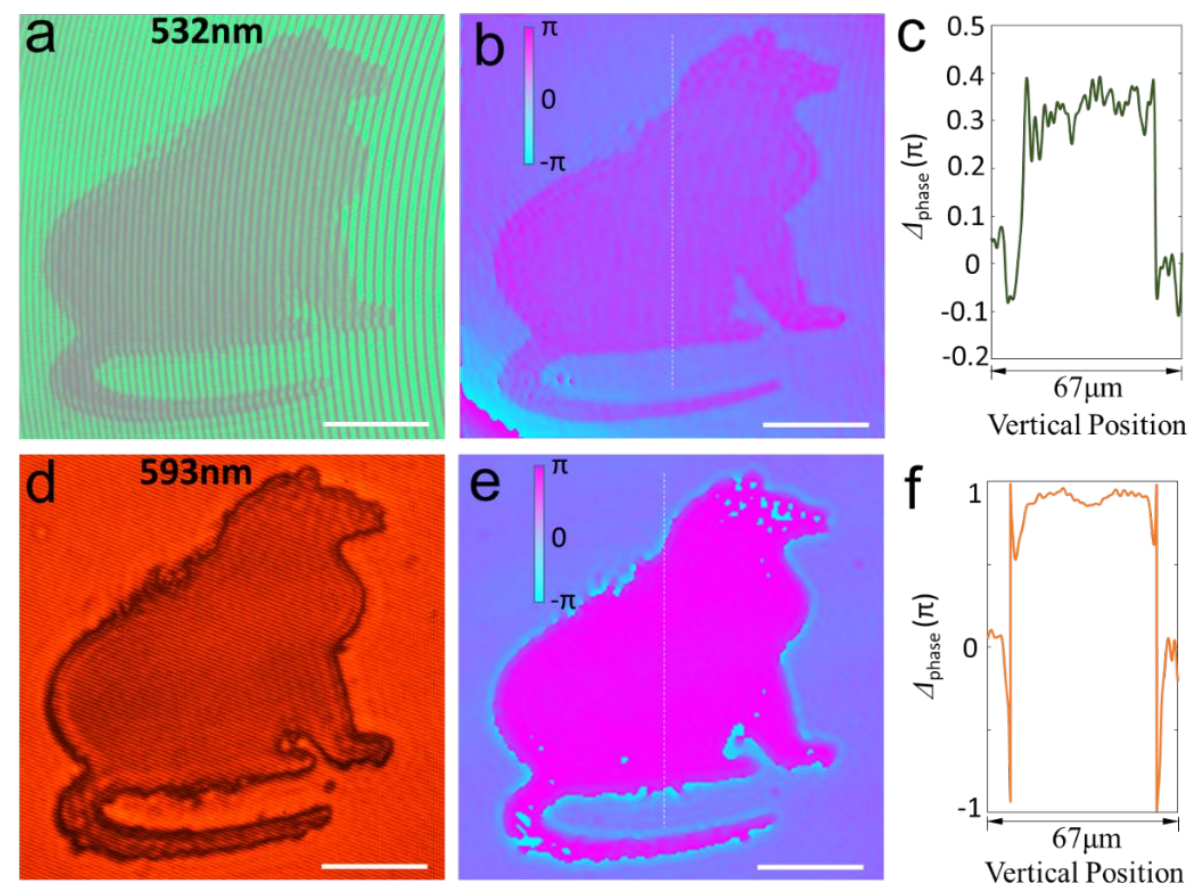

Figure S5. Experimental interference patterns, the retrieved phase and their linescanning profiles at the wavelengths of $532 \mathrm{~nm}(\mathrm{a}-\mathrm{c})$ and $593 \mathrm{~nm}(\mathrm{~b}-\mathrm{f})$. It reveals that the "gun" pattern in the "cat" is well-camouflaged, without any jump of transmission and phase at the interface between the "gun" and the meta-structures.
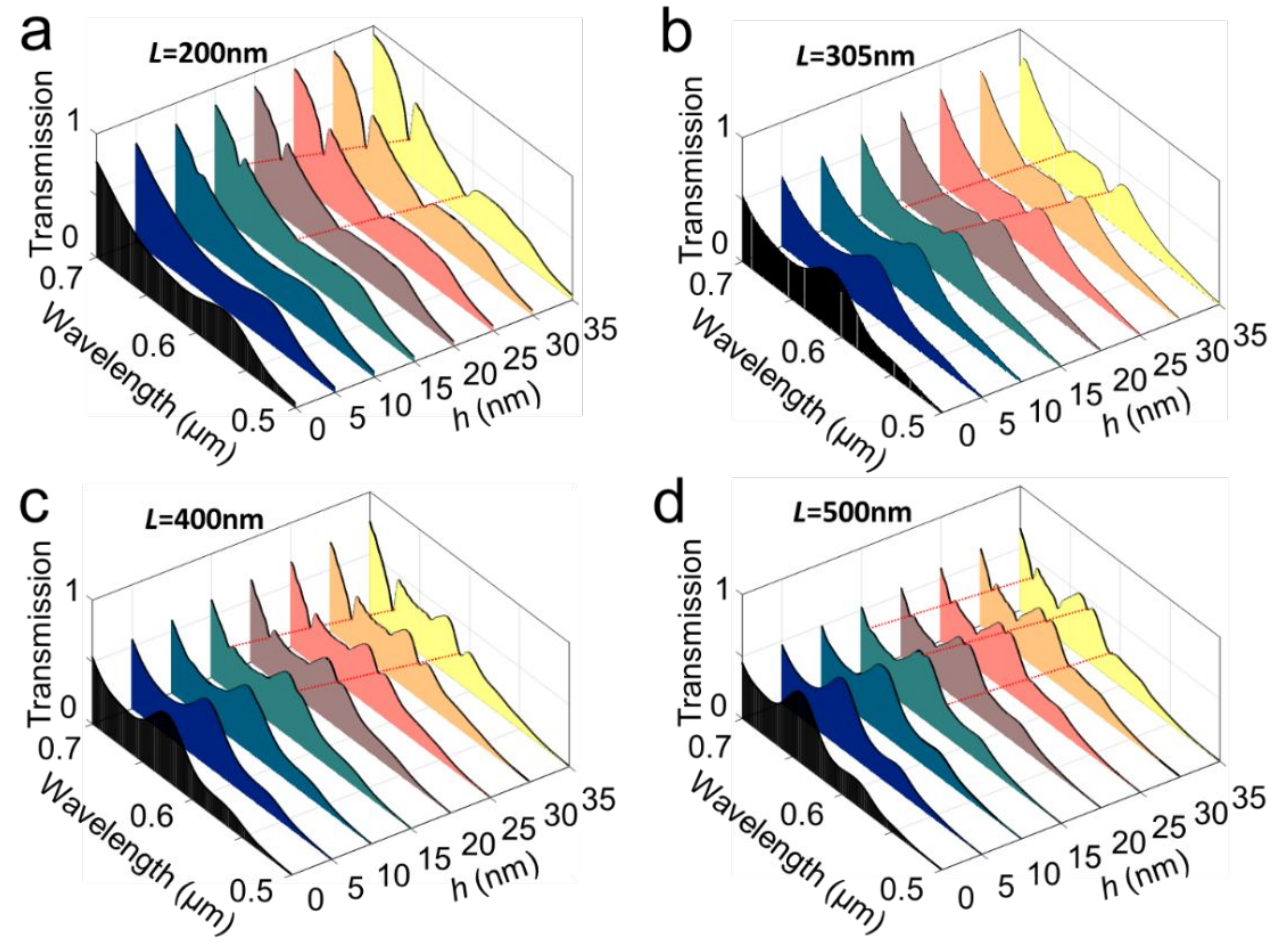

Figure S6. Simulated transmission of the meta-structures with different $L: 200 \mathbf{~ n m}$ (a), $305 \mathrm{~nm}$ (b), $400 \mathrm{~nm}$ (c) and $500 \mathrm{~nm}$ (d). All the simulations show that the nanomodes begin to appear at $h=15 \mathrm{~nm}$, where the valleys at the transmission spectrum are obvious. It implies that the $h_{\max }$ is fixed for a given material. 

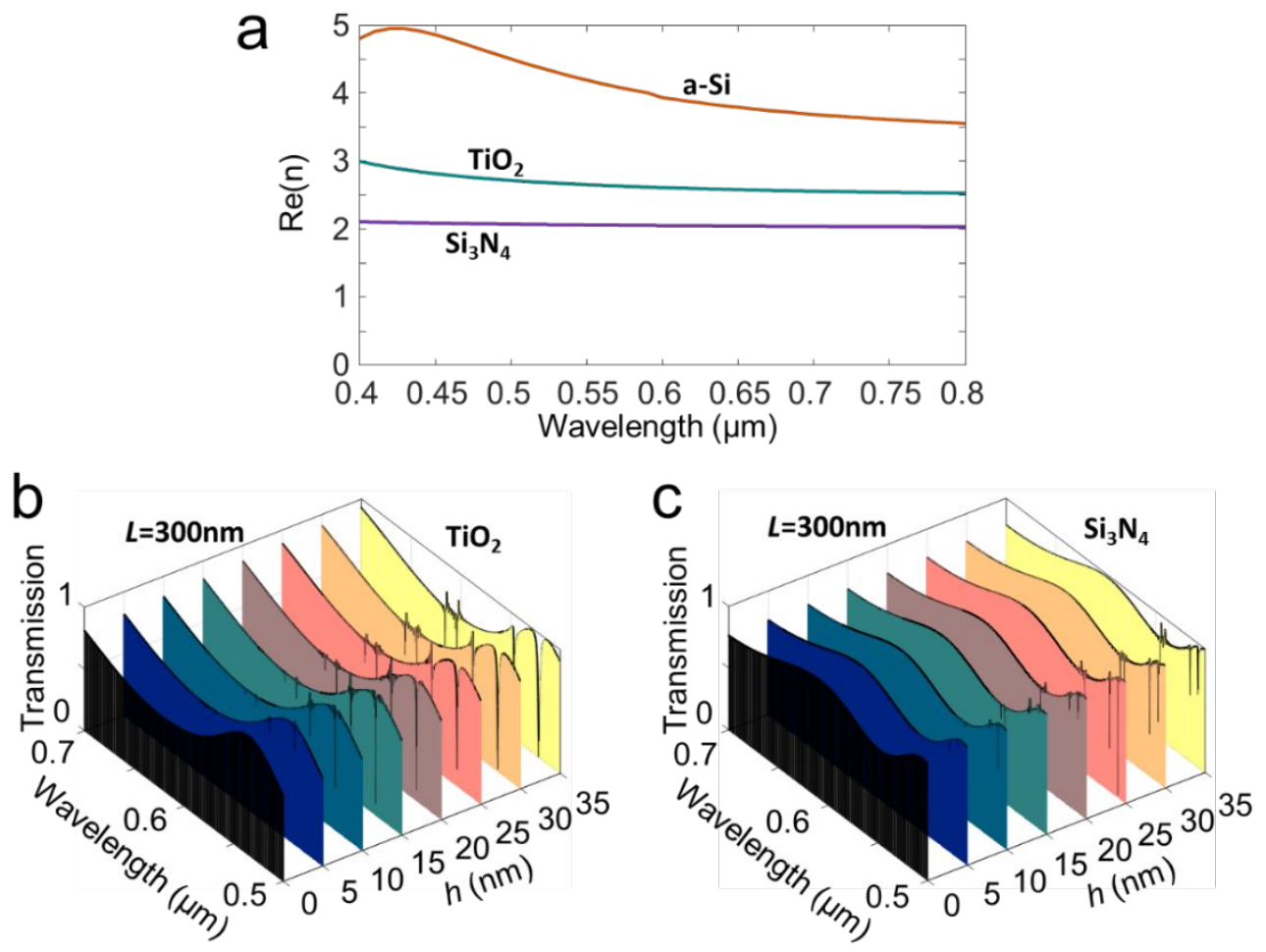

Figure S7. Simulated transmission of the meta-structures with different materials: $\mathrm{TiO}_{2}$ (b) and $\mathrm{Si}_{3} \mathrm{~N}_{4}(\mathrm{c})$, whose refractive indices are shown in (a) for a comparison. One can find that the $h_{\max }$ is equal to $20 \mathrm{~nm}$ for $\mathrm{TiO}_{2}$ and $35 \mathrm{~nm}$ for $\mathrm{Si}_{3} \mathrm{~N}_{4}$, which increases for the low-index material. It is contributed to the fact that the volume of nanomodes increase because of the increment of the equivalent wavelength in the material of low refractive index. In this application of optical masquerade, the nanomodes should be suppressed to keep the same transmission with the film. However, we note that the high-order nanomodes can be excited easily with a small $h$ (35 nm for the case $\mathrm{TiO}_{2}$ ), which, alternatively, is quite useful in nano-lasing and the enhancement of nonlinear signals and therefore deserves further investigation of this proposed metastructure. 
a
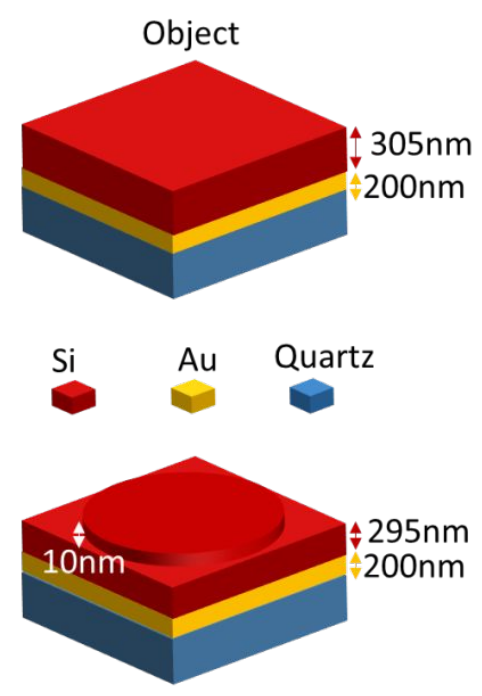

Perturbative Metasurfaces
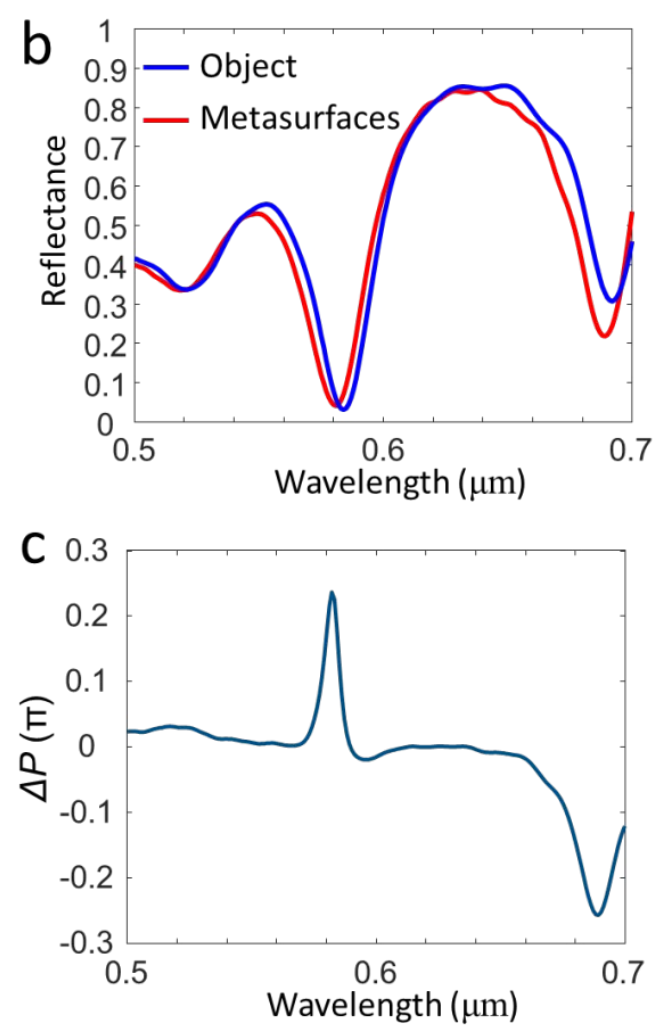

Figure S8. A proposed photonic masquerade in reflection mode. (a) A 200-nm-thick Au layer is designed to be placed between silicon and substrate in reflection mode, where the silicon film of object is also $305 \mathrm{~nm}$. The thickness of nano-disk array is optimized to be $10 \mathrm{~nm}$, leaving the 295nm-thick bottom layer. By using FDTD method, the simulated reflectance spectra (b) for both objects and masquerade metasurfaces are very close. The difference (c) between their phase profile is trivial $(<0.25 \pi)$. It confirms that our proposed masquerade could work in a reflective mode. 